\title{
3. ¿Cómo se sospecha una fibrosis pulmonar idiopática en la atención primaria de salud?
}

\author{
RENÉ FABA B.*, RICARDO TOBAR H.**, VALESKA GLASINOVIC B.*** y JAVIER CERDA C.****
}

\section{How is idiopathic pulmonary fibrosis suspected in primary health care?}

Idiopathic pulmonary fibrosis (IPF) is a lung disease of nonspecific symptomatology. Progressive chronic cough and dyspnea in older smokers or ex-smokers are the most frequent symptoms and clinical features. Therefore, it is usual for the diagnosis to be late. Primary care constitutes the patient's first contact with the health system. Therefore, it is necessary to deliver all possible information to physicians, nurses and physical therapists who care for adults with respiratory problems, since IPF will only be diagnosed if FPI is considered.

Key words: Idiopathic pulmonary fibrosis; Cough; Dyspnea; Primary Care Health.

\section{Resumen}

La fibrosis pulmonar idiopática (FPI) es una enfermedad pulmonar de sintomatología inespecífica. La tos crónica y disnea progresivas en adultos mayores fumadores o exfumadores son los sintomas y características clínicas más frecuentes. Por lo tanto, es usual que el diagnóstico sea tardío. La atención primaria constituye el primer contacto del paciente con el sistema de salud. Por esto es necesaria la entrega de toda la información posible a los médicos, enfermeras y kinesiólogos que atienden adultos con problemas respiratorios, ya que sólo se diagnosticará FPI si se piensa en FPI.

Palabras clave: Fibrosis pulmonar idiopática; tos; disnea; cuidados de atención primaria.

La fibrosis pulmonar idiopática (FPI) es una enfermedad pulmonar de sintomatología inespecífica, que se manifiesta fundamentalmente mediante tos crónica y disnea progresiva, afecta principalmente a pacientes sobre los 60 años. Por lo tanto, es frecuente que el diagnóstico sea tardío, ya que se confunde con múltiples patologías con síntomas semejantes y de mayor prevalencia.

La atención primaria constituye el primer contacto del paciente con el sistema de salud. Por eso, es necesaria la entrega de toda la información posible a los médicos, enfermeras y kinesiólogos que atienden adultos con problemas respiratorios subagudos y crónicos, ya que solo se diagnosticará FPI si se piensa en FPI. En la práctica, suelen estudiarse y tratarse a los pacientes con FPI como personas con insuficiencia cardía- ca, asma bronquial o enfermedad pulmonar obstructiva crónica (EPOC). Esto se traduce en un retraso en el diagnóstico y derivación a atención especializada en una media de 1,5 a 2 años ${ }^{1,2}$.

La sospecha de que un paciente pueda estar cursando una FPI debe surgir de las siguientes condiciones en historia, examen físico, espirometría o radiografía de tórax ${ }^{3}$ :

- Típicamente en adultos de más 60 años, aunque la sospecha debe iniciarse desde los 40 años.

- Tos seca por más de 8 semanas, en general, refractaria a tratamientos habituales.

- Disnea de esfuerzo persistente y progresiva en los últimos 3 meses, sin otra explicación evidente.

- Neumonías recurrentes en el adulto mayor.

* Clínica Indisa. Santiago, Chile.

** Hospital San Borja Arriarán. Santiago, Chile.

*** Clínica Indisa. Red Salud Vitacura.Santiago, Chile.

**** Hospital Dipreca: Dirección de Previsión de Carabineros de Chile. Santiago, Chile. 
- Antecedentes familiares de fibrosis pulmonar.

- Crepitaciones secas inspiratorias, basales bilaterales tipo "velcro" que persisten después de varias respiraciones profundas y que no se modifican con la tos.

- Hipocratismo digital.

- Caída significativa (más de 4 puntos) de la saturación de oxígeno medida por oximetría de pulso frente a un esfuerzo físico realizado en propio consultorio (mini-caminata).

- Espirometría con limitación ventilatoria restrictiva (aunque puede ser normal).

- Patrón de enfermedad pulmonar intersticial en radiografía o tomografía de tórax.

Todos los pacientes bajo sospecha de una enfermedad pulmonar intersticial difusa deben ser derivados a los Consultorios Adosados de Especialidades (CAE) de hospitales Tipo I y 2 (alta complejidad) para establecer el diagnóstico, según los protocolos definidos.

\section{Bibliografía}

1.- PUROKIVI M, HODGSON U, MYLLÄRNIEMI M, SALOMAA ER, KAARTEENAHO R. Are physicians in primary health care able to recognize pulmonary fibrosis? Eur Clin Respir J 2017; 4: 1290339. DOI: 10. 1080/20018525.2017.1290339.

2.- MOLINA J, TRIGUEROS JA, QUINTANO JA, MASCARÓS E, XAUBET A, ANCOCHEA J. Fibrosis pulmonar idiopática: un reto para la atención primaria. Semergen 2014; 40: 134-42.

3.- ZIBRAK JD, PRICE D. Interstitial lung disease: raising the index of suspicion in primary care. NPJ Prim Care Respir Med 2014; 24: 14054. doi: 10.1038/ npjpcrm.2014.54.
Correspondencia a:

Dr. René Faba B.

Email: renefaba@gmail.com 